\title{
Diversidade e equitabilidade de Plantas Alimentícias Não Convencionais na zona rural de Viçosa, Minas Gerais, Brasil
}

\author{
BARREIRA, T.F.1; PAULA FILHO, G.X.1*; RODRIGUES, V.C.C. ${ }^{1 ;}$ ANDRADE, F.M.C.'; SANTOS, R.H.S.1; \\ PRIORE, S.E.'; PINHEIRO-SANT'ANA, H.M.'. \\ 1Universidade Federal de Viçosa (UFV), Programa de Pós-Graduação em Agroecologia, Av. PH Rolfs, Viçosa, \\ MG, CEP: 36570-900. *Autor para correspondência: galdinoxpf@gmail.com.
}

\begin{abstract}
RESUMO: O presente estudo analisou a diversidade e equitabilidade de plantas alimentícias não convencionais (PANCs) na zona rural de Viçosa, Minas Gerais, Brasil. A pesquisa foi conduzida utilizando a amostragem Bola de Neve com entrevistas semiestruturadas aplicadas à 20 moradores de nove comunidades rurais. Avaliou-se a frequência relativa de citação das espécies (Fr); a diversidade e equitabilidade das mesmas, utilizando os índices de ShannonWiener ( $\left.\mathrm{H}^{\prime}\right)$ e de Pielou (J'), respectivamente. As espécies foram listadas pela família, nome científico, nomes populares, ambiente de propagação, hábito de crescimento, formas de consumo, estado de domesticação, ciclo de produção e registro por meio de consultas em herbários do bioma Mata Atlântica. Foram encontradas 59 espécies de PANCs, distribuídas em 30 famílias botânicas e 48 gêneros. As famílias asteraceae e myrtaceae se destacaram pela riqueza florística, contribuindo com 11 e 7 espécies, respectivamente. Espécies da família asteraceae obtiveram as maiores frequências relativas (32,2). Obteve-se índice de diversidade Shannon-Wiener de 1,65 (Base 10), e de equitabilidade de Pielou de 0,93. Os resultados encontrados estão similares à de outros estudos desenvolvidos no bioma Mata Atlântica, observou-se ampla diversidade de PANCs na área de estudo, e o conhecimento sobre estas encontra-se distribuído uniformemente entre os moradores.
\end{abstract}

Palavras-chave: PANCs, etnobotânica, conhecimento popular, prospecção de plantas alimentícias, recursos alimentares vegetais.

\begin{abstract}
Diversity and equivalence of unconventional food plants in rural zone of Viçosa, Minas Gerais, Brazil. This study analyzed the diversity and the equity of unconventional food plants (UFPs) in the rural area of Viçosa, Minas Gerais, Brazil. The research was performed using the Snowball sampling with semi-structured interviews, applied to 20 residents from nine rural communities. We evaluated the relative frequency of citation of the species (Fr); the diversity and equity of the same species, using the Shannon-Wiener $\left(\mathrm{H}^{\prime}\right)$ and Pielou $\left(\mathrm{J}^{\prime}\right)$ indexes, respectively. The species were listed by its family, scientific name, common denominations, propagation environment, growth habit, application methods, domestication state, production cycle and finally registration through consultation in herbaria in the Atlantic Forest biome. We found 59 species of UFPs, distributed in 30 botanical families and 48 genera. The Asteraceae family and the Myrtaceae one stood out for their floristic richness, contributing to 11 and 7 species, respectively. Species from the Asteraceae family got the highest relative frequencies (32.2). It was obtained the Shannon-Wiener diversity index of 1.65 (Base 10), and the Pielou equity index of 0.93 . The results are similar to other studies developed in the Atlantic Forest biome, where there was a wide diversity of UFPs in the study area, and the knowledge of these plants is evenly distributed among the residents.
\end{abstract}

Keywords: UFPs, ethnobotany, popular knowledge, food plants prospecting, plant food resources.

\section{INTRODUÇÃO}

A utilização de plantas como recursos alimentícios pelo homem ocorre desde os tempos pré-históricos; além da finalidade alimentícia, são utilizadas para fins medicinais, de construção e combustão (Nascimento et al., 2012; Nascimento et al., 2013). A utilização das plantas com finalidade 
alimentícia representa alternativa de subsistência para comunidades rurais e, podem contribuir com a economia local e regional (Nesbitt et al., 2010). A utilização de plantas alimentícias, em particular as PANCs, é parte da cultura, identidade e práticas agrícolas em muitas regiões do planeta (Voggesser et al., 2013).

As PANCs estão entre as fontes de alimentos que se desenvolvem em ambientes naturais sem a necessidade de insumos e da derrubada de novas áreas (Bressan et al., 2011). O fato de muitas dessas plantas estarem em áreas manejadas por agricultores torna-se estratégia fundamental para o fortalecimento da soberania alimentar de muitas famílias (Cruz-Garcia \& Price, 2011). Entretanto, muitas dessas plantas, embora disponíveis a baixo custo, ainda são desconhecidas e subutilizadas por uma parcela significativa da população (Kinupp, 2007; Luizza et al., 2013). Somase à essa realidade, a publicidade dos fastfood, que contribuem para a adoção de novos hábitos alimentares e para a perda da soberania alimentar de muitas famílias (King et al., 2011).

O consumo das PANCs pode ser estratégia para manter a diversificação alimentar, estimulando a manutenção da floresta. Se realizado de maneira sustentável, pode ser considerada uma forma de utilização com baixo impacto na agricultura, associada à conservação ambiental (Kinupp, 2007). As PANCs estão presentes em determinadas comunidades ou regiões, onde ainda exercem influência na alimentação de populações tradicionais, porém passaram a ter expressão econômica e social reduzidas, perdendo espaço para outros produtos (Brasil, 2010).

No Brasil, diversas PANCs são utilizadas para consumo alimentar de muitas famílias, sendo as mesmas consumidas in natura, refogadas, em formas de doces, cocadas, dentre outros; porém, ainda são poucos os estudos sobre o uso destas plantas (Crepaldi et al., 2001; Albuquerque \& Andrade, 2002; Kinupp \& Barros, 2008; Miranda \& Hanazaki, 2008; Pilla \& Amorozo, 2009; Nascimento et al., 2012; Nascimento et al., 2013).

Esse potencial ainda desconhecido exige a realização de mais pesquisas, e pode se tornar ferramenta importante no estabelecimento de sistemas de produção em bases sustentáveis, uma vez que esses recursos ainda são consumidos por parte da população rural e estão adaptadas às condições edafoclimáticas de muitas regiões (Brasil, 2010).

A partir destas considerações, o objetivo deste trabalho foi registrar a diversidade, formas de uso, ambiente de propagação, hábito de crescimento, estado de domesticação, ciclo de produção e identificação botânica de PANCs encontradas na zona rural de Viçosa, Minas Gerais. Para avaliar a diversidade das espécies e a distribuição local do conhecimento sobre as mesmas, foi analisada a frequência relativa de citação (Fr) e foram determinados os índices diversidade de Shannon-Wiener $\left(\mathrm{H}^{\prime}\right)$, e de equitabilidade Pielou (J'), respectivamente. Embora, encontrem-se poucos estudos sobre este tema, a presente investigação pode contribuir para o aprofundamento de estudos sobre a diversidade de PANCs, por meio de métodos científicos já consolidados na literatura acadêmica e que podem ser reproduzidos em outras situações de pesquisa.

\section{MATERIAL E MÉTODOS}

\section{Área de estudo}

O presente estudo foi realizado no período de outubro a dezembro de 2012, nas comunidades rurais: Buieié, Estação Velha, Palmital, Silêncio, Violeira, Zig-zag, Córrego Fundo, Cachoeirinha e São José do Triunfo, no município de Viçosa, Minas Gerais, Brasil, situado no domínio morfoclimático da Mata Atlântica (Figura 1). Em relação aos aspectos de agricultura e extrativismo, na zona rural de Viçosa encontra-se produção de café (Coffea canéfora L.), horticultura e pequenos animais. Nas comunidades estudadas são utilizados recursos alimentares e plantas medicinais pelas populações locais (Barreira, 2013). Essas comunidades são habitadas por nativos da região, com origem na zona rural, tendo a agricultura como forma de sobrevivência, algumas famílias utilizam recursos da floresta para fins alimentícios e medicinais, sendo esta prática herdada dos ancestrais e estando diretamente relacionada com princípios filosóficos e religiosos. Entre as comunidades estudadas, encontram-se remanescentes quilombolas, com semelhanças relacionadas à alimentação, religião, lazer e outros traços culturais (Barreira, 2013).

\section{Amostragem dos entrevistados}

Para chegar aos entrevistados utilizou-se a técnica de rede, conhecida na antropologia como "Network" e nas ciências sociais, como amostragem não probabilística, definida por Patton (1990), Cotton (1996) e Pinheiro (2003) como "Amostragem Bola de Neve" ("snow ball"). O critério de amostragem para inclusão dos entrevistados deu-se a partir da identificação de pessoas nas comunidades rurais, reconhecidas como consumidoras de PANCs. Após estabelecer contato, foi realizada entrevista semiestruturada por meio de roteiro com perguntas préelaboradas. Uma vez finalizada a entrevista, pediase que o entrevistado indicasse uma nova pessoa, também conhecedora das PANCs. O processo foi

Rev. Bras. PI. Med., Campinas, v.17, n.4, supl. II, p.964-974, 2015. 


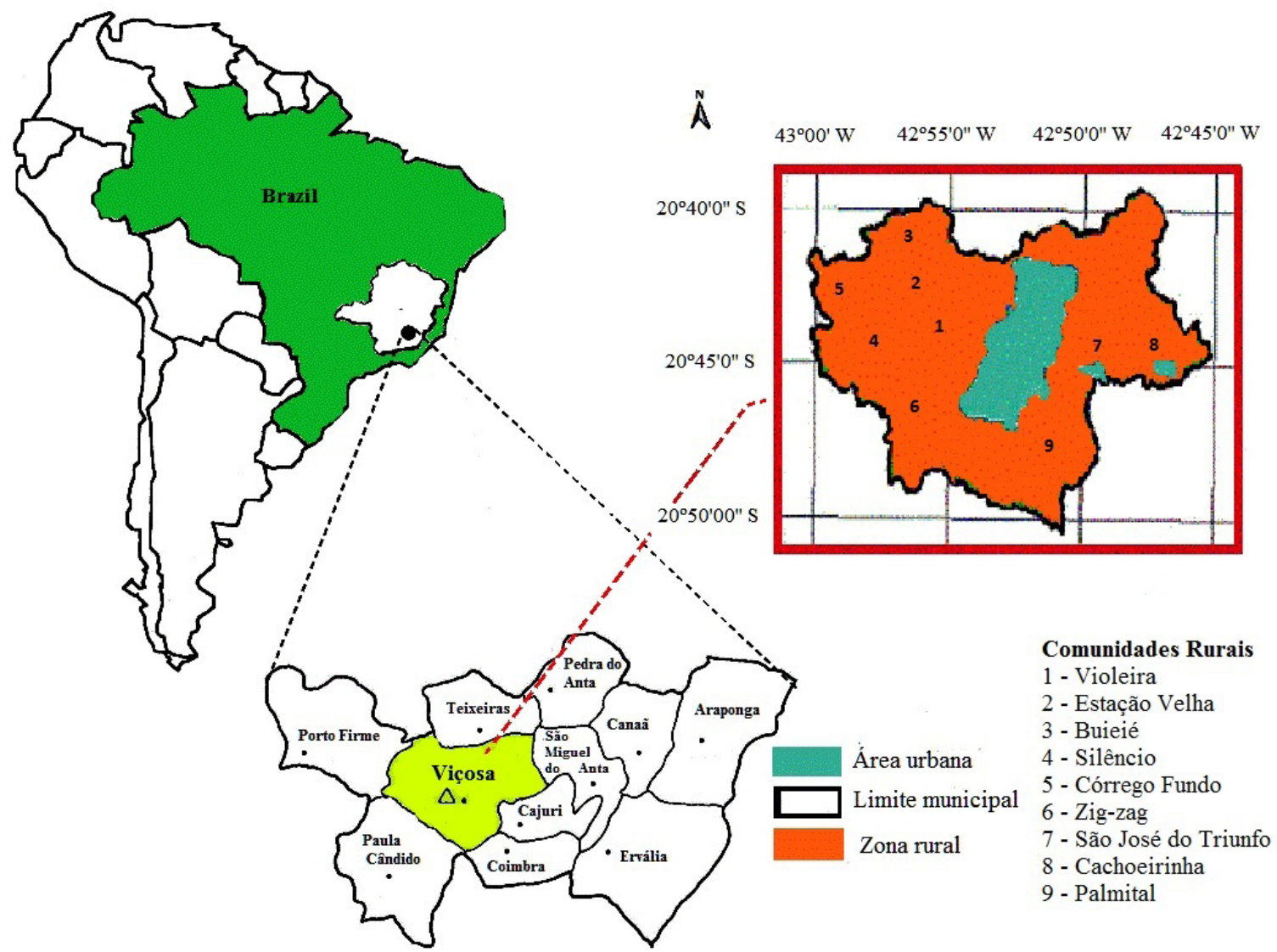

FIGURA 1. Área de estudo. Zona rural do município de Viçosa, Estado de Minas Gerais, Brasil.

se repetindo a partir de novos incluídos, conforme metodologia preconizada por Patton (1990), Cotton (1996) e Pinheiro (2003), de forma que as pessoas conhecedoras de PANCs foram validadas pela própria comunidade.

\section{Obtenção dos dados de campo}

Após estabelecer contato com os informantes-chave, foram realizadas entrevistas semiestruturadas por meio de roteiro com perguntas pré-elaboradas sobre as PANCs consumidas pela família e suas formas de uso. Informações sobre a caracterização física da planta e do ambiente foram obtidas por meio de ficha para coleta de material botânico. Uma vez finalizada a entrevista, pedia-se que o entrevistado indicasse nova pessoa, também conhecedora de PANCs e o processo foi se repetindo a partir de novos incluídos. O roteiro de entrevistas foi testado previamente com grupo de quatro famílias. As perguntas foram realizadas de forma oral e individualmente aos informanteschave em seus domicílios e durante o percurso pela propriedade. Quando havia tendência à estabilização do número de espécies diferentes de plantas citadas, e o número de espécies não se alterava substancialmente, encerrava-se a pesquisa (Ming, 1995).
A coleta do material botânico foi realizada junto aos entrevistados, no momento das entrevistas e em visitas posteriores. Este procedimento, conhecido por "turnê guiada" foi utilizado visando evitar erros na identificação, advindos dos nomes populares repetidos para algumas plantas (Albuquerque \& Lucena, 2004). Além do informante citar a espécie, o mesmo a apontou "in loco", conforme sugerem Albuquerque et al., (2010).

As espécies foram fotografadas em ambiente natural, coletadas e identificadas quanto às formas de uso e características botânicas.

As espécies foram listadas pela família, nome científico, nomes populares. As categorias referentes ao ambiente de propagação (em meio à cultura agrícola, horta, fragmento florestal, pasto, brejo e pomar), hábito de crescimento (herbáceo, árvore, arbustivo e liana), estado de domesticação (coletada e cultivada), ciclo de produção (anual e perene) foram determinadas de acordo com Lorenzi (1992). As formas de uso (refogadas em molhos e caldos, in natura, endosperma líquido, polpa, amêndoa, empanada, doces, compotas) foram organizadas de acordo com base em Kinupp (2007).

Registro e identificação botânica das espécies foram realizados por meio de comparação com amostras do acervo do herbário da Universidade 
Federal de Viçosa (VIC), além de outros herbários (RB, IAC, OUPR, EAC, UESC, ESA, HPL, BHCB, $\mathrm{K}$, HSJRP, INPA, MAC) que detém espécies encontradas no Bioma Mata Atlântica; e por meio de pesquisas nas seguintes bibliografias especializadas: Lorenzi, (1992); Lorenzi \& Matos, (2008); Lorenzi et al., (2006).

\section{Sistematização dos dados}

Para a classificação das espécies em famílias foi adotado o sistema Angiosperm Phylogeny Group III (APG III, 2009). A nomenclatura das espécies e abreviações dos autores foi realizada por meio de informações disponíveis na Base de Dados Trópicos, do Missouri Botanical Garden (Trópicos, 2013).

A frequência relativa de citação $(F r)$ das espécies foi determinada de acordo com Begossi (1996).

A diversidade das espécies de PANCs foi obtido por meio do índice de Shannon-Wiener $\left(\mathrm{H}^{\prime}\right)$, de acordo com Begossi (1996).

A distribuição do conhecimento sobre as espécies de PANCs entre os moradores das comunidades (informantes-chave) foi obtida por meio do índice de equitabilidade Pielou (J') segundo a metodologia proposta por Magurran (1988).

Os índices foram calculados com o auxílio do software Mata Nativa ${ }^{\circledR}$ (Cientec, 2010).

\section{Aspectos éticos}

O estudo foi aprovado pelo Comitê de Ética em Pesquisa com Seres Humanos da UFV (Ref. No 121/2012/CEPH/wmt). Os entrevistados foram informados dos objetivos do estudo e do sigilo das informações e assinaram o Termo de Consentimento Livre e Esclarecido, sendo a liberdade do consentimento em participar da pesquisa garantida a todos, conforme preconizado pelas Diretrizes e Normas Regulamentadoras de Pesquisa envolvendo Seres Humanos do Conselho Nacional de Saúde (Brasil, 1996).

\section{RESULTADOS}

Foram visitadas nove comunidades rurais e entrevistados 20 informantes-chave, sendo 12 homens e oito mulheres.

Entre os informantes, 12 são naturais de Viçosa, sete são de municípios circunvizinhos, e apenas um é de outra região do estado, mas reside em Viçosa há 19 anos. No geral, $80 \%$ de todos os informantes residem no município há mais de 30 anos.

A faixa etária dos informantes $(n=20)$ variou entre 43 e 93 anos, sendo a maioria (75\%) composta por idosos, acima de 65 anos.
Foram identificadas 59 espécies de PANCs com potencial alimentício, distribuídas em 30 famílias e 48 gêneros botânicos, totalizando 389 citações. A família mais citada foi a Asteraceae, que representou $28,5 \%$ do total de citações, seguida pela Myrtaceae com 9,5\%.

$\mathrm{Na}$ tabela 1 encontram-se as informações sobre as PANCs encontradas no presente estudo.

As famílias asteraceae e myrtaceae apresentaram o maior número de espécies de PANCs encontradas, sendo 11 e sete espécies, respectivamente.

Quanto ao ambiente de propagação, as PANCs foram encontradas em pastagens $(27 \%)$, no meio de culturas agrícolas $(24 \%)$ e em hortas domésticas (22\%). Mais da metade destas apresentam hábito de crescimento herbáceo (57\%), sendo, portanto de menor porte. Apresentam ciclos produtivos curtos, porém se propagam com mais facilidade, sendo encontradas principalmente dispersas ao longo de pastagens, em meio à culturas agrícolas e em hortas domésticas.

As espécies de PANCs são consumidas principalmente de forma refogada (cozida) em molhos e caldos (47\%) e in natura (40\%). Embora sejam espécies não convencionais, algumas dessas $(27 \%)$ se encontram domesticadas e cultivadas em meio à hortas, pomares ou outras culturas agrícolas; enquanto que as demais $(73 \%)$ são coletadas principalmente em pastos, fragmentos florestais e também em meio à culturas agrícolas.

Há relação entre o ciclo de produção das PANCs identificadas e os hábitos de crescimento das mesmas, as que desenvolvem ciclos perenes predominantemente apresentam hábito de crescimento como árvores e arbustivos, enquanto que aquelas com hábito de crescimento herbáceas e lianas apresentam ciclos anuais e perenes ao mesmo tempo. As PANCs com ciclo de produção anual em sua maioria são herbáceas.

A frequência relativa de citação das 59 espécies de PANCs encontradas no presente estudo encontra-se relacionada na tabela abaixo.

Estudos etnobotânicos têm utilizado cálculos de índices de diversidade $\left(H^{\prime}\right)$ para avaliar a diversidade do conhecimento sobre recursos vegetais. No presente estudo o valor de H' foi igual a 1,65.

O grau de equitabilidade $\left(J^{\prime}\right)$ varia numa escala de $0 \leq J^{\prime} \leq 1$, quanto mais próximo de 1 , significa que o conhecimento está melhor distribuído entre os comunitários. No presente estudo obtevese $J^{\prime}=0,93$.

\section{DISCUSSÃO}

Observou-se correlação entre o

Rev. Bras. PI. Med., Campinas, v.17, n.4, supl. II, p.964-974, 2015. 
TABELA 1. Família botânica, nome científico, nome popular, ambiente de propagação, hábito de crescimento, formas de consumo, estado de domesticação e registro nos herbários, de PANCs encontradas na zona rural do município de Viçosa, Minas Gerais, 2013.

\begin{tabular}{|c|c|c|c|c|c|c|c|c|}
\hline $\begin{array}{l}\text { Família } \\
\text { botânica }\end{array}$ & Nome científico & Nome popular & $\begin{array}{l}\text { Ambiente de } \\
\text { propagação }\end{array}$ & $\begin{array}{l}\text { Hábito de } \\
\text { crescimento }\end{array}$ & $\begin{array}{l}\text { Formas de } \\
\text { consumo }\end{array}$ & $\begin{array}{l}\text { Estado de } \\
\text { domesticação }\end{array}$ & Ciclo & Registro \\
\hline Amarantaceae & $\begin{array}{l}\text { Amaranthus } \\
\text { hybridus L. }\end{array}$ & Caruru & $\begin{array}{l}\text { Em meio à cultura } \\
\text { agrícola }\end{array}$ & Herbáceo & $\begin{array}{l}\text { Refogada em } \\
\text { molhos e caldos }\end{array}$ & Coletada & Anual & RB 00524769 \\
\hline Annonaceae & $\begin{array}{l}\text { Annona sylvatica } \\
\text { Dunal }\end{array}$ & $\begin{array}{l}\text { Araticum do } \\
\text { mato }\end{array}$ & $\begin{array}{l}\text { Fragmento } \\
\text { florestal }\end{array}$ & Árvore & In natura & Coletada & Perene & VIC 35535 \\
\hline \multirow{3}{*}{ Arecaceae } & $\begin{array}{l}\text { Astrocaryum } \\
\text { aculeatissimum } \\
\text { (Schott) }\end{array}$ & $\begin{array}{l}\text { Coco de } \\
\text { brejaúva }\end{array}$ & Pasto & Árvore & $\begin{array}{l}\text { Endosperma } \\
\text { líquido e amêndoa }\end{array}$ & Coletada & Perene & VIC 5764 \\
\hline & Attalea dubia Mart. & Coco indaiá & Pasto & Árvore & Polpa e amêndoa & Coletada & Perene & VIC 20234 \\
\hline & $\begin{array}{l}\text { Syagrus coronata } \\
\text { (Mart.) Becc. }\end{array}$ & Coco Licuri & Pasto & Árvore & Polpa e amêndoa & Coletada & Perene & VIC 7120 \\
\hline \multirow{11}{*}{ Asteraceae } & $\begin{array}{l}\text { Erechtites } \\
\text { valerianaefolia L. }\end{array}$ & Capiçova & Pasto & Herbáceo & $\begin{array}{l}\text { Refogada em } \\
\text { molhos e caldos }\end{array}$ & Coletada & Anual & IAC 39256 \\
\hline & $\begin{array}{l}\text { Taraxacum officinale } \\
\text { Weber. }\end{array}$ & Dente de leão & Horta & Herbáceo & $\begin{array}{l}\text { Refogada em } \\
\text { molhos e caldos }\end{array}$ & Coletada & Anual & IAC 4209 \\
\hline & Emilia sonchifolia L. & Serralhinha & $\begin{array}{l}\text { Em meio à cultura } \\
\text { agrícola }\end{array}$ & Herbáceo & $\begin{array}{l}\text { Refogada em } \\
\text { molhos e caldos }\end{array}$ & Coletada & Anual & IAC 29393 \\
\hline & Bidens pilosa L. & Picão & Pasto & Herbáceo & $\begin{array}{l}\text { Refogada em } \\
\text { molhos e caldos }\end{array}$ & Coletada & Anual & IAC 12956 \\
\hline & $\begin{array}{l}\text { Sonchus oleraceus } \\
\text { L. }\end{array}$ & $\begin{array}{l}\text { Serralha do } \\
\text { mato }\end{array}$ & Pasto & Herbáceo & $\begin{array}{l}\text { Refogada em } \\
\text { molhos e caldos }\end{array}$ & Coletada & Anual & IAC 6669 \\
\hline & Sonchus arvensis L. & Serralha lisa & Horta & Herbáceo & $\begin{array}{l}\text { Refogada em } \\
\text { molhos e caldos }\end{array}$ & Cultivada & Anual & RB 00428940 \\
\hline & Sonchus asper L. & $\begin{array}{l}\text { Serralha } \\
\text { espinhenta }\end{array}$ & Pasto & Herbáceo & $\begin{array}{l}\text { Refogada em } \\
\text { molhos e caldos }\end{array}$ & Coletada & Anual & IAC 6668 \\
\hline & Cichorium intybus L. & $\begin{array}{l}\text { Almeirão de } \\
\text { árvore }\end{array}$ & Horta & Herbáceo & $\begin{array}{l}\text { Refogada em } \\
\text { molhos e caldos }\end{array}$ & Cultivada & Anual & IAC 21696 \\
\hline & $\begin{array}{l}\text { Hypochoeris } \\
\text { brasiliensis Griseb. }\end{array}$ & $\begin{array}{l}\text { Almeirão do } \\
\text { mato }\end{array}$ & Pasto & Herbáceo & $\begin{array}{l}\text { Refogada em } \\
\text { molhos e caldos }\end{array}$ & Coletada & Anual & OUPR 14942 \\
\hline & $\begin{array}{l}\text { Lactuca canadensis } \\
\mathrm{L} \text {. }\end{array}$ & Almeirão roxo & Horta & Herbáceo & $\begin{array}{l}\text { Refogada em } \\
\text { molhos e caldos }\end{array}$ & Cultivada & Anual & IAC 1095 \\
\hline & $\begin{array}{l}\text { Vernonanthura } \\
\text { patens (Kunth) }\end{array}$ & Cambará & Pasto & Arbustivo & Empanada & Coletada & Perene & RB 00548953 \\
\hline \multirow{2}{*}{ Basellaceae } & Basella rubra L. & Bertalha & Horta & Herbáceo & $\begin{array}{l}\text { Refogada em } \\
\text { molhos e caldos }\end{array}$ & Cultivada & Perene & EAC 27657 \\
\hline & $\begin{array}{l}\text { Anredera cordifolia } \\
\text { (Ten.) Steenis. }\end{array}$ & $\begin{array}{l}\text { Lobrobô } \\
\text { verdadeiro }\end{array}$ & Horta & Herbáceo & $\begin{array}{l}\text { Refogada em } \\
\text { molhos e caldos }\end{array}$ & Cultivada & Anual & RB 00052112 \\
\hline Bromeliaceae & $\begin{array}{l}\text { Ananas bracteatus } \\
\text { (Lindl.), var. albus }\end{array}$ & Ananás do mato & $\begin{array}{l}\text { Fragmento } \\
\text { florestal }\end{array}$ & Arbustivo & In natura & Coletada & Perene & VIC 6310 \\
\hline \multirow{3}{*}{ Cactaceae } & $\begin{array}{l}\text { Pereskia aculeata } \\
\text { Mill. }\end{array}$ & Lobrobô miúdo & Horta & Herbáceo & $\begin{array}{l}\text { Refogada em } \\
\text { molhos e caldos }\end{array}$ & Cultivada & Perene & RB 00659585 \\
\hline & $\begin{array}{l}\text { Pereskia grandiflora } \\
\text { Haw. }\end{array}$ & Lobrobô graúdo & Pasto & Herbáceo & $\begin{array}{l}\text { Refogada em } \\
\text { molhos e caldos }\end{array}$ & Coletada & Perene & UESC 16183 \\
\hline & $\begin{array}{l}\text { Pereskia bahiensis } \\
\text { Gurke. }\end{array}$ & $\begin{array}{l}\text { Ora-pró-nobis } \\
\text { ereta }\end{array}$ & Horta & Herbáceo & $\begin{array}{l}\text { Refogada em } \\
\text { molhos e caldos }\end{array}$ & Cultivada & Perene & RB 00064731 \\
\hline Comelinaceae & $\begin{array}{l}\text { Tradescantia } \\
\text { fluminensis Vell. }\end{array}$ & Trapoeraba & Horta & Herbáceo & $\begin{array}{l}\text { Refogada em } \\
\text { molhos e caldos }\end{array}$ & Coletada & Anual & RB 00730853 \\
\hline Convolvulaceae & Ipomoea batata L. & $\begin{array}{l}\text { Folha de batata- } \\
\text { doce }\end{array}$ & $\begin{array}{l}\text { Em meio à cultura } \\
\text { agrícola }\end{array}$ & Herbáceo & $\begin{array}{l}\text { Refogada em } \\
\text { molhos e caldos }\end{array}$ & Cultivada & Anual & RB 00262102 \\
\hline \multirow[t]{2}{*}{ Cruciferae } & $\begin{array}{l}\text { Lepidium } \\
\text { pseudodidymum } \\
\text { Thell. ex Druce } \\
\end{array}$ & Mentrasto & $\begin{array}{l}\text { Em meio à cultura } \\
\text { agrícola }\end{array}$ & Herbáceo & $\begin{array}{l}\text { Refogada em } \\
\text { molhos e caldos }\end{array}$ & Coletada & Anual & ESA 5439 \\
\hline & Sinapis arvensis L. & $\begin{array}{l}\text { Mostarda } \\
\text { silvestre }\end{array}$ & $\begin{array}{l}\text { Em meio à cultura } \\
\text { agrícola }\end{array}$ & Herbáceo & $\begin{array}{l}\text { Refogada em } \\
\text { molhos e caldos }\end{array}$ & Coletada & Anual & RB 00072942 \\
\hline Clusiaceae & $\begin{array}{l}\text { Rheedia gardneriana } \\
\text { Planch. \& Triana }\end{array}$ & Bacupari & $\begin{array}{l}\text { Fragmento } \\
\text { florestal }\end{array}$ & Árvore & In natura & Coletada & Perene & VIC 5825 \\
\hline \multirow{2}{*}{ Cucurbitaceae } & $\begin{array}{l}\text { Sicana odorifera } \\
\text { Naud. }\end{array}$ & Maracujina & Pomar & Liana & In natura e suco & Cultivada & Perene & VIC 20283 \\
\hline & $\begin{array}{l}\text { Sicana sphaerica } \\
\text { Vell. }\end{array}$ & Melão croá & Pomar & Liana & In natura e suco & Cultivada & Perene & VIC 7491 \\
\hline Euphorbiaceae & $\begin{array}{l}\text { Manihot esculenta } \\
\text { Crantz. }\end{array}$ & $\begin{array}{l}\text { Folha de } \\
\text { mandioca }\end{array}$ & $\begin{array}{l}\text { Em meio à cultura } \\
\text { agrícola }\end{array}$ & Herbáceo & $\begin{array}{l}\text { Refogada em } \\
\text { molhos e caldos }\end{array}$ & Cultivada & Anual & RB 00086736 \\
\hline
\end{tabular}


TABELA 1. Família botânica, nome científico, nome popular, ambiente de propagação, hábito de crescimento, formas de consumo, estado de domesticação e registro nos herbários, de PANCs encontradas na zona rural do município de Viçosa, Minas Gerais, 2013.

...continuação

\begin{tabular}{|c|c|c|c|c|c|c|c|c|}
\hline Fabaceae & Inga vera Willd. & Ingá banana & Pasto & Árvore & In natura & Coletada & Perene & VIC 40933 \\
\hline Lamiaceae & Stachys lanata L. & Peixinho & Horta & Herbáceo & Empanada & Cultivada & Perene & HPL 3643 \\
\hline Malvaceae & $\begin{array}{l}\text { Hibiscus sabdariffa } \\
\text { L. }\end{array}$ & Vinagreira & Horta & Arbustivo & Doces e compotas & Cultivada & Anual & RB 00573974 \\
\hline \multirow{7}{*}{ Myrtaceae } & $\begin{array}{l}\text { Psidium } \\
\text { guineense Sw. }\end{array}$ & Araçá do campo & Pasto & Arbustivo & In natura & Coletada & Perene & VIC 10594 \\
\hline & $\begin{array}{l}\text { Psidium } \\
\text { cattleyanum Sabine }\end{array}$ & Araçá vermelho & Pasto & Arbustivo & In natura & Coletada & Perene & VIC 31253 \\
\hline & Plinia edulis (Vell.) & Cambucá & $\begin{array}{l}\text { Fragmento } \\
\text { florestal }\end{array}$ & Árvore & In natura e suco & Coletada & Perene & VIC 9614 \\
\hline & $\begin{array}{l}\text { Campomanesia } \\
\text { pubescens Berg. }\end{array}$ & $\begin{array}{l}\text { Gabiroba do } \\
\text { mato }\end{array}$ & $\begin{array}{l}\text { Fragmento } \\
\text { florestal }\end{array}$ & Arbustivo & $\begin{array}{l}\text { In natura, suco e } \\
\text { doces }\end{array}$ & Coletada & Perene & VIC 9729 \\
\hline & $\begin{array}{l}\text { Eugenia myrtifolia } \\
\text { L. }\end{array}$ & $\begin{array}{l}\text { Laranjinha do } \\
\text { mato }\end{array}$ & $\begin{array}{l}\text { Fragmento } \\
\text { florestal }\end{array}$ & Árvore & In natura & Coletada & Perene & VIC 11944 \\
\hline & Syzygium cumini L. & Jambolão & $\begin{array}{l}\text { Fragmento } \\
\text { florestal }\end{array}$ & Árvore & In natura & Coletada & Perene & VIC 9875 \\
\hline & Eugenia uniflora L. & Pitanga & $\begin{array}{l}\text { Fragmento } \\
\text { florestal }\end{array}$ & Árvore & In natura & Coletada & Perene & VIC 7732 \\
\hline Oxalidaceae & $\begin{array}{l}\text { Oxalis corniculata } \\
\text { L. }\end{array}$ & Trevo azedo & Horta & Herbáceo & In natura & Coletada & Anual & RB 00270849 \\
\hline \multirow[b]{2}{*}{ Passifloraceae } & Passiflora vitifolia L. & Maracujá mirim & Pomar & Liana & In natura e suco & Coletada & Perene & VIC 6534 \\
\hline & $\begin{array}{l}\text { Passiflora } \\
\text { amethystina J. C. } \\
\text { Mikan }\end{array}$ & $\begin{array}{l}\text { Maracujá do } \\
\text { mato }\end{array}$ & Pomar & Liana & In natura e suco & Coletada & Perene & VIC 2426 \\
\hline Piperaceae & Piper jaborandi Vell. & Jaborandi & $\begin{array}{l}\text { Em meio à cultura } \\
\text { agrícola }\end{array}$ & Herbáceo & Empanada & Coletada & Perene & ВНСВ 72260 \\
\hline \multirow{2}{*}{ Plantaginaceae } & Plantago major L. & $\begin{array}{l}\text { Transagem da } \\
\text { horta }\end{array}$ & $\begin{array}{l}\text { Em meio à cultura } \\
\text { agrícola }\end{array}$ & Herbáceo & $\begin{array}{l}\text { Refogada em } \\
\text { molhos e caldos }\end{array}$ & Cultivada & Anual & UESC 12160 \\
\hline & $\begin{array}{l}\text { Plantago coriacea } \\
\text { Cham. \& Schltdl. }\end{array}$ & $\begin{array}{l}\text { Transagem do } \\
\text { mato }\end{array}$ & $\begin{array}{l}\text { Em meio à cultura } \\
\text { agrícola }\end{array}$ & Herbáceo & $\begin{array}{l}\text { Refogada em } \\
\text { molhos e caldos }\end{array}$ & Coletada & Anual & K 000573569 \\
\hline Polygalaceae & $\begin{array}{l}\text { Diclidanthera } \\
\text { elliptica Miers. }\end{array}$ & $\begin{array}{l}\text { Jabuticaba de } \\
\text { rama }\end{array}$ & $\begin{array}{l}\text { Fragmento } \\
\text { florestal }\end{array}$ & Liana & In natura & Coletada & Perene & VIC 13203 \\
\hline Polygonaceae & Rumex acetosa L. & Azedinha/labaça & $\begin{array}{l}\text { Em meio à cultura } \\
\text { agrícola }\end{array}$ & Herbáceo & In natura & Cultivada & Perene & IAC 48845 \\
\hline Polypodiaceae & $\begin{array}{l}\text { Pteridium } \\
\text { aquillinum } \mathrm{L} .\end{array}$ & $\begin{array}{l}\text { Samambaia do } \\
\text { mato }\end{array}$ & Pasto & Herbáceo & $\begin{array}{l}\text { Refogada em } \\
\text { molhos e caldos }\end{array}$ & Coletada & Anual & HSJRP 07661 \\
\hline Portulacacea & $\begin{array}{l}\text { Portulaca oleracea } \\
\text { L. }\end{array}$ & Beldroega & $\begin{array}{l}\text { Em meio à cultura } \\
\text { agrícola }\end{array}$ & Herbáceo & In natura & Coletada & Anual & INPA 111808 \\
\hline Rosaceae & $\begin{array}{l}\text { Rubus rosifolius } \\
\text { Smith. }\end{array}$ & Amora do mato & $\begin{array}{l}\text { Fragmento } \\
\text { florestal }\end{array}$ & Arbustivo & In natura & Coletada & Perene & VIC 3856 \\
\hline \multirow{4}{*}{ Solanaceae } & $\begin{array}{l}\text { Lycopersicon } \\
\text { esculentum Mill }\end{array}$ & Folha de tomate & Horta & Herbáceo & $\begin{array}{l}\text { Refogada em } \\
\text { molhos e caldos }\end{array}$ & Cultivada & Anual & ESA 927 \\
\hline & Solanum juciri Mart. & Jequiri & Pomar & Liana & In natura & Cultivada & Anual & VIC 8517 \\
\hline & Physalis angulata L. & $\begin{array}{l}\text { Juá manso (Juá } \\
\text { Pocã) }\end{array}$ & Pasto & Arbustivo & In natura & Coletada & Perene & VIC 5924 \\
\hline & $\begin{array}{l}\text { Solanum nigrum Var. } \\
\text { americanum Mill. }\end{array}$ & Erva moura & Pasto & Arbustivo & In natura & Coletada & Perene & VIC 4111 \\
\hline Tropaeolaceae & Tropaeolum majus L. & . Capuchinha & $\begin{array}{l}\text { Em meio à cultura } \\
\text { agrícola }\end{array}$ & Herbáceo & In natura & Cultivada & Anual & ESA 16766 \\
\hline Typhaceae & Typha angustifolia L. & Taboa & Brejo & Herbáceo & $\begin{array}{l}\text { Refogada em } \\
\text { molhos e caldos }\end{array}$ & Coletada & Anual & ESA 82541 \\
\hline \multirow{2}{*}{ Urticaceae } & Fleurya aestuans L. & Cansanção & $\begin{array}{l}\text { Em meio à cultura } \\
\text { agrícola }\end{array}$ & Herbáceo & $\begin{array}{l}\text { Refogada em } \\
\text { molhos e caldos }\end{array}$ & Coletada & Anual & MAC 325 \\
\hline & Boehmeria nivea L. & Rami & $\begin{array}{l}\text { Em meio à cultura } \\
\text { agrícola }\end{array}$ & Herbáceo & $\begin{array}{l}\text { Refogada em } \\
\text { molhos e caldos }\end{array}$ & Cultivada & Anual & ESA 6117 \\
\hline
\end{tabular}

Rev. Bras. Pl. Med., Campinas, v.17, n.4, supl. II, p.964-974, 2015. 
TABELA 2. Frequência relativa de citação $(F e)$ das espécies de PANCs encontradas na zona rural do município de Viçosa, Minas Gerais, 2013.

\begin{tabular}{|c|c|c|c|c|}
\hline Nome popular & Família botânica & Nome científico & Citações & $(\mathrm{Fe})^{*}$ \\
\hline Capiçova & Asteraceae & Erechtites valerianaefolia L. & 19 & 32,20 \\
\hline Serralha do mato & Asteraceae & Sonchus oleraceus L. & 19 & 32,20 \\
\hline Lobrobô miúdo & Cactaceae & Pereskia aculeata Mill. & 19 & 32,20 \\
\hline Serralha lisa & Asteraceae & Sonchus arvensis L. & 16 & 27,12 \\
\hline Seralha espinhenta & Asteraceae & Sonchus asper L. & 16 & 27,12 \\
\hline Mostarda silvestre & Cruciferae & Sinapis arvensis L. & 15 & 25,42 \\
\hline Taboa & Typhaceae & Typha angustifolia L. & 14 & 23,73 \\
\hline Jabuticaba de rama & Polygalaceae & Diclidanthera elliptica Miers. & 13 & 22,03 \\
\hline Almeirão do mato & Asteraceae & Hypochoeris brasiliensis Griseb. & 12 & 20,34 \\
\hline Maracujá mirim & Passifloraceae & Passiflora vitifolia L. & 12 & 20,34 \\
\hline Almeirão roxo & Asteraceae & Lactuca canadensis L. & 11 & 18,64 \\
\hline Maracujá do mato & Passifloraceae & Passiflora amethystina J. C. Mikan & 11 & 18,64 \\
\hline Samambaia do mato & Polypodiaceae & Pteridium aquillinum $\mathrm{L}$. & 11 & 18,64 \\
\hline Araçá do campo & Myrtaceae & Psidium guineense Sw. & 10 & 16,95 \\
\hline Amora do mato & Rosaceae & Rubus rosifolius Smith. & 10 & 16,95 \\
\hline Jequiri & Solanaceae & Solanum juciri Mart. & 10 & 16,95 \\
\hline Araticum do mato & Annonaceae & Annona sylvatica Dunal & 9 & 15,25 \\
\hline Almeirão de árvore & Asteraceae & Cichorium intybus $\mathrm{L}$. & 9 & 15,25 \\
\hline Lobrobô graúdo & Cactaceae & Pereskia grandiflora Haw. & 9 & 15,25 \\
\hline Ingá banana & Fabaceae & Inga vera Willd. & 8 & 13,56 \\
\hline Caruru & Amarantaceae & Amaranthus hybridus L. & 7 & 11,86 \\
\hline Coco Licuri & Arecaceae & Syagrus coronata (Mart.) Becc. & 7 & 11,86 \\
\hline Ananás do mato & Bromeliaceae & Ananas bracteatus (Lindl.), var. albus & 7 & 11,86 \\
\hline Maracujina & Cucurbitaceae & Sicana odorifera Naud. & 7 & 11,86 \\
\hline Bacupari & Clusiaceae & Rheedia gardneriana Planch. \& Triana & 6 & 10,17 \\
\hline Mentrasto & Cruciferae & Lepidium pseudodidymum Thell. ex Druce & 6 & 10,17 \\
\hline Araçá vermelho & Myrtaceae & Psidium cattleyanum Sabine & 6 & 10,17 \\
\hline Cambucá & Myrtaceae & Plinia edulis (Vell.) & 6 & 10,17 \\
\hline Juá manso & Solanaceae & Physalis angulata $\mathrm{L}$. & 6 & 10,17 \\
\hline Melão croá & Cucurbitaceae & Sicana sphaerica Vell. & 5 & 8,47 \\
\hline Jambolão & Myrtaceae & Syzygium cumini L. & 5 & 8,47 \\
\hline Erva moura & Solanaceae & Solanum nigrum Var. americanum Mill. & 5 & 8,47 \\
\hline Capuchinha & Tropaeolaceae & Tropaeolum majus L. & 5 & 8,47 \\
\hline Cambará & Asteraceae & Vernonanthura patens (Kunth) & 4 & 6,78 \\
\hline Folha de batata-doce & Convolvulaceae & Ipomoea batata $L$. & 4 & 6,78 \\
\hline Gabiroba do mato & Myrtaceae & Campomanesia pubescens Berg. & 4 & 6,78 \\
\hline Pitanga & Myrtaceae & Eugenia uniflora L. & 4 & 6,78 \\
\hline Lobrobô verdadeiro & Basellaceae & Anredera cordifolia (Ten.) Steenis. & 3 & 5,08 \\
\hline Trevo azedo & Oxalidaceae & Oxalis corniculata L. & 3 & 5,08 \\
\hline Coco de brejaúva & Arecaceae & Astrocaryum aculeatissimum (Schott) & 3 & 5,08 \\
\hline Azedinha/labaça & Polygonaceae & Rumex acetosa L. & 3 & 5,08 \\
\hline Beldroega & Portulacacea & Portulaca oleracea L. & 3 & 5,08 \\
\hline Coco indaiá & Arecaceae & Attalea dúbia Mart. & 2 & 3,39 \\
\hline Serralhinha & Asteraceae & Emilia sonchifolia L. & 2 & 3,39 \\
\hline
\end{tabular}

Rev. Bras. PI. Med., Campinas, v.17, n.4, supl. II, p.964-974, 2015. 
TABELA 2. Frequência relativa de citação (Fe) das espécies de PANCs encontradas na zona rural do município de Viçosa, Minas Gerais, 2013.

\begin{tabular}{lllll}
\hline Picão & & & 2 & 3,39 \\
\hline Bertalha & Asteraceae & Bidens pilosa L. & 2 & 3,39 \\
\hline Peixinho & Basellaceae & Basella rubra L. & 2 & 3,39 \\
\hline Laranjinha do mato & Lamiaceae & Stachys lanata $L$. & 2 & 3,39 \\
\hline Jaborandi & Myrtaceae & Eugenia myrtifolia L. & 2 & 3,39 \\
\hline Transagem da horta & Piperaceae & Piper jaborandi Vell. & 2 & 3,39 \\
\hline Transagem do mato & Plantaginaceae & Plantago coriacea Cham. \& Schltdl. & 2 & 3,39 \\
\hline Cansanção & Urticaceae & Fleurya aestuans L. & 2 & 3,39 \\
\hline Dente de leão & Asteraceae & Taraxacum officinale Weber. & 1 & 1,69 \\
\hline Ora-pró-nobis ereta & Cactaceae & Pereskia bahiensis Gurke. & 1 & 1,69 \\
\hline Trapoeraba & Comelinaceae & Tradescantia fluminensis Vell. & 1,69 \\
\hline Folha de mandioca & Euphorbiaceae & Manihot esculenta Crantz. & 1 & 1,69 \\
\hline Vinagreira & Malvaceae & Hibiscus sabdariffa L. & 1 & 1,69 \\
\hline Folha de tomate & Solanaceae & Lycopersicon esculentum Mill & 1 & 1,69 \\
\hline Rami & Urticaceae & Boehmeria nivea L. & 1 \\
\hline
\end{tabular}

* $\mathrm{Fr}=$ Resultado obtido de acordo com Begossi (1996), a partir do número de citações da espécie, multiplicado por 100, dividido pelo numero de espécies de PANCs encontradas.

conhecimento dos informantes e o tempo de residência na mesma comunidade, visto que $80 \%$ dos informantes residem há mais de 30 anos na região de estudo. Esta relação está de acordo ao observado por Maldonado et al., (2013) em estudo realizado no México, no qual analisaram o valor de uso e a importância ecológica da flora silvestre por indígenas; neste trabalho, os autores concluíram que o conhecimento e uso dos recursos da flora foi maior entre os nativos, que residiam há mais tempo na comunidade, do que entre populações mestiças que eventualmente estavam se deslocando, mesmo entre comunidades próximas.

A faixa etária dos informantes revela que os idosos são os maiores conhecedores das formas de uso, épocas de frutificação, ambiente de propagação, dentre outras utilidades das PANCs. Estas também foram as conclusões observadas por Hanazaki et al., (2000) e Borges \& Peixoto (2009) em estudo realizado sobre o conhecimento e uso de plantas em comunidade caiçara no Rio de Janeiro, ambos estudos revelam que, de maneira geral, os mais idosos conhecem uma diversidade maior de plantas úteis, cujo saber foi acumulado ao longo de sua vivência e contato com os mesmos

A metodologia empregada no presente trabalho alcançou resultados que mostram que as gerações mais novas dispõem de menor conhecimento sobre as espécies de PANCs. Ademais, o êxodo de muitos desses jovens para a zona urbana por motivos diversos (trabalho, estudo e outros), pode ser um fator limitante para o fluxo do conhecimento sobre os recursos vegetais alimentares conforme já observado por Amorozo (2002).

As famílias botânicas asteraceae e myrtaceae se destacaram no presente estudo, mostrando sua importância alimentícia para as comunidades rurais estudadas, porém vale à pena frisar que as mesmas não fazem parte do grupo de alimentos mais consumidos no Brasil (Souza et al., 2013); reforçando o que Kinupp \& Barros (2008) já haviam observado em estudo similar, onde nota-se a desvalorização de espécies de plantas alimentícias nativas, em detrimento de espécies exóticas, que geralmente são mais caras, onerando os custos com alimentação e atingindo a renda de muitas famílias. Com base nestas observações, é possível inferir que as PANCs podem exercer importante estratégia no aspecto da soberania alimentar de muitas famílias, principalmente as rurais.

Observou-se que algumas espécies de PANCs são encontradas em ambientes de culturas agrícolas, principalmente em meio à cultura do café. Porém, a utilização de agrotóxicos em meio à estas culturas foi apontada como uma das causas da supressão de algumas espécies de PANCs, o que torna-se um agravante, visto que, além da supressão da espécie alimentícia, as que sobrevivem e que são coletadas nesse ambiente, podem conter o risco de contaminação à quem as consome.

Em relação à predominância das famílias asteraceae e myrtaceae dentre as espécies identificadas no presente estudo, estes resultados estão similares aos encontrados por Kinupp \& Barros (2007), em estudo realizado sobre a 
diversidade de PANCs na Região Metropolitana de Porto Alegre, onde estas famílias botânicas foram as que apresentaram o maior número de espécies encontradas. Miranda \& Hanazaki (2008) em estudo etnobotânico sobre o conhecimento e uso de recursos vegetais em comunidades de restinga em São Paulo e Santa Catarina também identificaram estas duas famílias com o maior número de espécies.

Mesmo em ambiente heterogêneo e alterado pela ação antrópica, como é a zona rural do município de Viçosa, as PANCs medram em meio à outros tipos de culturas agrícolas, pastos, fragmentos de florestas e beiras de estradas. Estes resultados corroboram a tese de Ramos-Zapata et al., (2013) ao afirmarem que as PANCs são espécies ruderais, desenvolvem facilidades de adaptação, mesmo que este ambiente esteja em elevado estado de alteração e perturbação.

Sobre a frequência relativa de citação, alguns estudos sobre levantamento e diversidade etnobotânica (Coelho-Ferreira \& Silva, 2005; Martins et al., 2005; Balcazar, 2012) têm considerado apenas as espécies que atinjam índices acima de $5 \%$. Considerando estes mesmos critérios para o presente estudo, observa-se que 42 espécies de PANCs encontradas, ou $75 \%$ destas se enquadram nesses critérios, destacando-se Erechtites valerianaefolia L., Sonchus oleraceus L. e Pereskia aculeata Mill.

De acordo com Begossi (1996), a frequência relativa de citação indica o quanto que uma determinada espécie se destaca em relação ao conjunto das demais, esse índice mostra a importância que esta espécies exerce nas comunidades estudadas. No presente estudo verificou-se que a família asteraceae, além de apresentar um conjunto maior de espécies de PANCs, estas respectivas espécies, individualmente, são as que apresentam a maior frequência relativa, com destaque para Erechtites valerianaefolia L., Sonchus oleraceus L., Sonchus arvensis L., Sonchus asper L., Hypochoeris brasiliensis Griseb. e Lactuca canadensis L. ou seja, estas espécies além de existirem em abundancia, as mesmas são as mais conhecidas e podem ser as mais consumidas. Resultados idênticos ao do presente estudo foram encontrados por Carniello et al., (2010) em abordagem etnobotanica realizada em quintais florestais de Mirassol D'Oeste, Mato Grosso, onde as asteráceas também obtiveram maior frequência relativa de citação.

O índice de Shannon-wiener $\left(\mathrm{H}^{\prime}\right)$ encontrado no presente estudo $\left(H^{\prime}=1,65\right)$ mostrou-se próximo à outros estudos realizados sobre a diversidades de recursos alimentares vegetais no bioma Mata Atlântica. Miranda \& Hanazaki (2008), em estudo sobre o uso de recursos vegetais, encontraram valores de $H^{\prime}=1,83$ e $H^{\prime}=1,90$, em comunidades de restinga em São Paulo e em Santa Catarina, respectivamente. Pilla \& Amorozo (2009) encontraram valores de $H^{\prime}=1,98$ em estudo realizado sobre recursos vegetais alimentares em bairros rurais no Vale do Paraíba, São Paulo. Os resultados destes trabalhos mostram que nestes ambientes a diversidade, ou riqueza destes recursos vegetais, foi maior do que no presente estudo.

O grau de equitabilidade ( $\mathrm{J}^{\prime}$ ) encontrado no presente estudo $\left(J^{\prime}=0,93\right)$ indica que o conhecimento está homogeneamente distribuído entre os comunitários, este resultado está próximo aos valores encontrados por Pilla \& Amorozo (2009), no qual obtiveram valor de $J^{\prime}=0,91$, para um grupo de 23 informantes. Lima et al., (2012) encontraram valores de J' entre 0,87 e 0,90 para homens adultos em estudo realizado sobre diversidade e uso de plantas do Cerrado em comunidades de Geraizeiros no Norte de Minas Gerais. Estes resultados revelam que entre os informantes do presente estudo, o conhecimento sobre estes recursos alimentares vegetais é mais homogêneo, se encontra melhor distribuído em relação aos informantes dos trabalhos citados.

Observa-se que trabalhos sobre índice de diversidade e de equitabilidade de espécies vegetais alimentares e medicinais têm sido realizados com maior frequência em áreas protegidas, ao contrário do presente estudo que foi realizado em comunidades com intensa exploração agrícola, o que pode interferir nos resultados encontrados. Ademais, a diversidade de metodologias adotadas, dificulta a comparação entre os resultados, porém, os índices encontrados no presente estudo encontram-se próximos aos principais estudos sobre recursos alimentares vegetais realizados no bioma Mata Atlântica.

\section{CONCLUSÕES}

Foram identificadas 59 espécies de PANCs, que representou 30 famílias botânicas e 48 gêneros, sendo a família Asteraceae a mais representativa, totalizando $11(18,6 \%)$ das 59 espécies identificadas. Espécies dessa família também foram as que apresentaram as maiores frequências relativas de citação, indicando que as mesmas, além de existirem em maior diversidade, são mais popularmente conhecidas nas comunidades estudadas.

As PANCS identificadas no presente estudo apresentam hábito de crescimento predominantemente herbáceo $(57 \%)$, são consumidas principalmente de forma refogada (cozida) em molhos e caldos $(47 \%)$ e in natura $(40 \%)$, e apresentam ciclos de produção anuais e

Rev. Bras. PI. Med., Campinas, v.17, n.4, supl. II, p.964-974, 2015. 
perenes.

O índice de Shannon-Wiener $\left(H^{\prime}=1,65\right)$ indica que a zona rural de Viçosa, apresenta elevada diversidade de PANCs, sendo a maioria, cultivadas em hortas ou coletadas em meio à culturas agrícolas, pastos e fragmentos florestais.

O índice de equitabilidade $\left(J^{\prime}=0,93\right)$ permite inferir que o conhecimento sobre PANCs na zona rural de Viçosa, Minas Gerais, Gerais, está homogeneamente distribuído entre os informantes da pesquisa.

\section{AGRADECIMENTOS}

Os autores agradecem à FUNARBE, FAPEMIG, CNPq e CAPES pela concessão de bolsa e suporte financeiro para realização dessa pesquisa, e às famílias de agricultores da zona rural do município de Viçosa, Minas Gerais, pela disponibilidade em participar desse estudo.

\section{REFERÊNCIAS}

ALBUQUERQUE, U.P.; ANDRADE, L.D.H.C. Conhecimento botânico tradicional e conservação em uma área de caatinga no Estado de Pernambuco, Nordeste do Brasil. Acta Botânica Brasílica, v.16, n.3, p.273-85, 2002.

ALBUQUERQUE, U.P.; LUCENA, R.F.P. Seleção e escolha dos informantes. In: ALBUQUERQUE, U.P.; LUCENA, R.F.P. Métodos e técnicas na pesquisa etnobotânica. 1 ed. Recife: Livro Rápido, 2004. p.19-36.

ALBUQUERQUE, U.P.; LUCENA, R.F.P.; ALENCAR, N.L. Métodos e técnicas para coleta de dados etnobiológicos. In: ALBUQUERQUE, U.P.; LUCENA, R.F.P.; CUNHA, L.V.F.C. Métodos e técnicas na pesquisa etnobiológica e etnoecológica. 2 ed. Recife: NUPEA, 2010. p. 39-64.

AMOROZO, M.C.M. Agricultura tradicional, espaços de resistência e o prazer de plantar. In: ALBUQUERQUE, U.P.; ALVES, A.G.C.; BORGES, A.C.L.; SILVA, V.A. Atualidades em etnobiologia e etnoecologia. 1 ed. Recife: SBEE, 2002. p. 123-131.

APG III. An update of the Angiosperm Phylogeny Group classification for the orders and families of flowering plants: APG III. Botanical Journal of the Linnen a Society, v.161, n.1, p.105-21, 2009.

BALCAZAR, A.L. Hipótese da aparência na dinâmica do uso de plantas medicinais na Floresta Nacional do Araripe (Ceará, nordeste do Brasil). 2012. 83f. Dissertação (Mestrado - Área de concentração em Botânica) - Departamento de Botânica, Universidade Federal Rural de Pernambuco, Recife.

BARREIRA, T.F. Levantamento e investigação do valor nutricional de hortaliças não-convencionais na zona rural de Viçosa, MG. 2013. 94f. Dissertação (Mestrado - Área de concentração em Agroecologia) - Departamento de Nutrição e Saúde, Universidade Federal de Viçosa, Viçosa.

BEGOSSI, A. Use of ecological methods in ethnobotany: diversity indices. Economic botany, v.50, n.3, p.28089, 1996.

BORGES, R.; PEIXOTO, A.L. Conhecimento e uso de plantas em uma comunidade caiçara do litoral sul do Estado do Rio de Janeiro, Brasil. Acta Botânica Brasílica, v.23, n.3, p.769-79, 2009.

BRASIL. Diretrizes e normas regulamentadoras de pesquisa envolvendo seres humanos / Conselho Nacional de Saúde. Brasília: CNS, 1996. 20p.

BRASIL. Manual de hortaliças não convencionais / Ministério da Agricultura, Pecuária e Abastecimento. Brasília: MAPA, 2010. 92p.

BRESSAN, R.A. et al. Stress-adapted extremophiles provide energy without interference with food production. Food Security, v.3, n.1, p.93-105, 2011.

CARNIELLO, M.A. et al. Quintais urbanos de Mirassol D'Oeste-MT, Brasil: uma abordagem etnobotânica. Acta Amazônica, v.40, n.3, p.451-70, 2010.

CIENTEC. Mata Nativa 3: Manual do usuário. 1 ed. Viçosa: UFV, 2010. $126 \mathrm{p}$.

COELHO-FERREIRA, M.R.; SILVA, M.F.F. A Fitofarmacopéia da Comunidade Pesqueira de Marudá, Litoral Paraense. Boletim do Museu Paraense Emilio Goeldi, v.1, n.2, p.31-43, 2005.

COTTON, C. M. Ethnobotany: principles and applications. 1 ed. Chichester: John Wiley e Sons, 1996. 434 p.

CREPALDI, I.C. et al. Composição nutricional do fruto de licuri (Syagrus coronata (Martius) Beccari). Revista Brasileira de Botânica, v.24, n.2, p.155-59, 2001.

CRUZ-GARCIA, G.S.; PRICE, L.L. Ethnobotanical investigation of'wild'food plants used by rice farmers in Kalasin, Northeast Thailand. Journal of ethnobiology and ethnomedicine, v.7, n.33, p.1-20, 2011.

HANAZAKI, N. et al. Diversity of plant uses in two Caiçara communities from the Atlantic Forest coast, Brazil. Biodiversity and Conservation, v.9, n.5, p.597-615, 2000.

KING, L. et al. Industry self regulation of television food advertising: Responsible or responsive? International journal of pediatric obesity, v.6, n.2, p.390-98, 2011.

KINUPP, V.F. Plantas alimentícias não-convencionais da região metropolitana de Porto Alegre, RS. 2007. 590f. Tese (Doutorado - Área de concentração em Fitotecnia) - Departamento de Horticultura e Silvicultura, Universidade Federal do Rio Grande do Sul, Porto Alegre.

KINUPP, V.F.; BARROS, I.B.I.D. Riqueza de Plantas Alimentícias Não-Convencionais na Região Metropolitana de Porto Alegre, Rio Grande do Sul. Revista Brasileira de Biociências, v.5, n.1, p.63-65, 2007.

KINUPP, V.F.; BARROS, I.B.I.D. Teores de proteína e minerais de espécies nativas, potenciais hortaliças $e$ frutas. Ciência e Tecnologia de Alimentos, v.28, n.4, p.846-57, 2008.

LIMA, I.L.P. et al. Diversidade e uso de plantas do Cerrado em comunidade de Geraizeiros no norte do estado de Minas Gerais, Brasil. Acta Botânica Brasílica, v.26, n.3, p.675-84, 2012.

LORENZI, H. Árvores brasileiras: manual de identificação e cultivo de plantas arbóreas nativas do Brasil. 3 ed. Nova Odessa: Instituto Plantarum de Estudos da Flora, 1992. 352p.

Rev. Bras. PI. Med., Campinas, v.17, n.4, supl. II, p.964-974, 2015. 
LORENZI, H.; MATOS, F.J.A.M. Plantas Medicinais no Brasil: nativas e exóticas. 2.ed. Nova Odessa: Instituto Plantarum, 2008. 544p.

LORENZI, H.; SARTORI, S.F.; BACHER, L.B.; LACERDA, M.T.C. Frutas Brasileiras e Exóticas Cultivadas: de consumo in natura. 1.ed. Nova Odessa: Instituto Plantarum, 2006. 650p.

LUIZZA, M.W. et al. Local Knowledge of Plants and their uses among Women in the Bale Mountains, Ethiopia. Ethnobotany Research \& Applications, v.11, n.1, p.315-39, 2013.

MAGURRAN, A.E. Ecological diversity and its measurement. 1. ed. London: Croom Helm, 1988. 179p.

MALDONADO, B. et al. Relationship between Use Value and Ecological Importance of Floristic Resources of Seasonally Dry Tropical Forest in the Balsas River Basin, México. Economic botany, v.67, n.1, p.17-29, 2013.

MARTINS, A.G. et al. Levantamento etnobotânico de plantas medicinais, alimentares e tóxicas da Ilha do Combu, Município de Belém, Estado do Pará, Brasil. Revista Brasileira de Farmácia, v.86, n.1, p.21-30, 2005.

MING, L.C. Levantamento de plantas medicinais na reserva extrativista "Chico Mendes" - Acre. 1995. 180f. Tese (Doutorado - Área de concentração em Botânica) - Departamento de Botânica. Universidade Estadual Paulista "Júlio de Mesquita Filho", Botucatu.

MIRANDA, T.M.; HANAZAKI, N. Conhecimento e uso de recursos vegetais de restinga por comunidades das ilhas do Cardoso (SP) e de Santa Catarina (SC), Brasil. Acta Botânica Brasílica, v.22, n.1, p.203-15, 2008.

NASCIMENTO, V.T. et al. Knowledge and Use of Wild Food Plants in Areas of Dry Seasonal Forests in Brazil.
Ecology of Food and Nutrition, v.52, n.4, p.317-43, 2013.

NASCIMENTO, V.T. et al. Famine Foods of Brazil s Seasonal Dry Forests: Ethnobotanical and Nutritional Aspects. Economic Botany, v.66, n.1, p.22-34, 2012.

NESBITT, M. et al. Linking biodiversity, food and nutrition: The importance of plant identification and nomenclature. Journal of food composition and analysis, v.23, n.6, p.486-98, 2010.

PATTON, M. Q. Qualitative evaluation and research methods. 2 ed. Newbury Park: Sage Publications, 1990. 536 p.

PILLA, M.A.C.; AMOROZO, M. O conhecimento sobre os recursos vegetais alimentares em bairros rurais no Vale do Paraíba, SP, Brasil. Acta Botânica Brasílica, v.23, n.4, p.1190-1201, 2009.

PINHEIRO, C.U. Técnicas e métodos antropológicos aplicados na Etnobotânica. 1. ed. Belém: MPEG, 2003. 39p.

RAMOS-ZAPATA, J.A. et al. Ruderal plants: temporary hosts of arbuscular mycorrhizal fungi in traditional agricultural systems? Tropical and Subtropical Agroecosystems, v.16, n.3, p.399-406, 2013.

SOUZA, A.M. et al. Alimentos mais consumidos no Brasil: Inquérito Nacional de Alimentação 2008-2009. Revista de Saúde Pública, v.47, Supl 1, p.190-99, 2013.

TROPICOS. Trópicos.org. Missouri Botanical Garden. Disponível em <http://www.tropicos.org/NameSearch. aspx?>. Acesso em: 19 julho 2013.

VOGGESSER, G. et al. Cultural impacts to tribes from climate change influences on forests. Climatic change, v.120, n.3, p.615-26, 2013.

Rev. Bras. PI. Med., Campinas, v.17, n.4, supl. II, p.964-974, 2015. 